\title{
Vaccine Protection Against Acquisition of Neutralization- Resistant SIV Challenges in Rhesus Monkeys
}

\author{
Dan H. Barouch ${ }^{1,2,{ }^{*}}$, Jinyan Liu ${ }^{1}$, Hualin Li ${ }^{1}$, Lori F. Maxfield ${ }^{1}$, Peter Abbink ${ }^{1}$, Diana M. \\ Lynch $^{1}$, M. Justin lampietro ${ }^{1}$, Adam SanMiguel ${ }^{1}$, Michael S. Seaman ${ }^{1}$, Guido Ferrari ${ }^{3}$, \\ Donald N. Forthal ${ }^{4}$, Ilnour Ourmanov ${ }^{5}$, Vanessa M. Hirsch ${ }^{5}$, Angela Carville ${ }^{6}$, Keith G. \\ Mansfield $^{6}$, Donald Stablein ${ }^{7}$, Maria G. Pau ${ }^{8}$, Hanneke Schuitemaker ${ }^{8}$, Jerald C. Sadoff ${ }^{8}$, \\ Erik M. Billings ${ }^{9}$, Mangala Rao ${ }^{9}$, Merlin L. Robb ${ }^{9}$, Jerome H. Kim ${ }^{9}$, Mary A. Marovich ${ }^{9}$, Jaap \\ Goudsmit ${ }^{8,{ }^{* *}}$, and Nelson L. Michael ${ }^{9,{ }^{* *}}$
}

${ }^{1}$ Division of Vaccine Research, Beth Israel Deaconess Medical Center, Harvard Medical School, Boston, MA $02215{ }^{2}$ Ragon Institute of MGH, MIT, and Harvard, Boston, MA $02114{ }^{3}$ Duke University Medical Center, Durham, NC $27710^{4}$ University of California Irvine School of Medicine, Irvine, CA $92697{ }^{5}$ National Institute of Allergy and Infectious Diseases, Bethesda, MA 20892 ${ }^{6} \mathrm{New}$ England Primate Research Center, Southborough, MA $01772{ }^{7}$ EMMES Corporation, Rockville, MD $20850{ }^{8}$ Crucell Holland BV, 2301 CA, Leiden, The Netherlands ${ }^{9}$ U.S. Military HIV Research Program, Walter Reed Army Institute of Research, Rockville, MD 20850

\section{Summary}

Preclinical studies of HIV-1 vaccine candidates have typically shown post-infection virologic control, but protection against acquisition of infection has previously only been reported against neutralization-sensitive virus challenges ${ }^{1-3}$. Here we demonstrate vaccine protection against acquisition of fully heterologous, neutralization-resistant virus challenges in rhesus monkeys. Adenovirus/poxvirus and adenovirus/adenovirus vector-based vaccines expressing SIVsmE543 Gag, Pol, and Env antigens resulted in a $\geq 80 \%$ reduction in the per-exposure probability of infection ${ }^{4,5}$ against repetitive, intrarectal SIVmac251 challenges in rhesus monkeys. Protection against acquisition of infection exhibited distinct immunologic correlates as compared with postinfection virologic control and required the inclusion of Env in the vaccine regimen. These data demonstrate the first proof-of-concept that optimized HIV-1 vaccine candidates can block acquisition of stringent, heterologous, neutralization-resistant virus challenges in rhesus monkeys.

Users may view, print, copy, download and text and data- mine the content in such documents, for the purposes of academic research, subject always to the full Conditions of use: http://www.nature.com/authors/editorial_policies/license.html\#terms

*To whom correspondence should be addressed. dbarouch@bidmc.harvard.edu.

Equal contribution

Author Contributions

D.H.B., M.G.P., H.S., J.C.S., J.G., M.L.R., J.H.K., M.A.M., and N.L.M. designed the study and analyzed data. J.L., H.L., D.M.L., M.J.I., and A.S. performed the cellular immunogenicity assays. M.J.I., M.S.S., G.F., D.N.F., E.M.B., and M.R. performed the humoral immunogenicity assays. L.F.M., P.A., I.O., and V.M.H. prepared the vaccine constructs. A.C. and K.G.M. led the clinical care of the rhesus monkeys. D.S. led the statistical analyses. D.H.B. led the study and wrote the paper with all co-authors.

Author Information

The authors declare no competing financial interests. M.G.P., H.S., J.C.S., and J.G. are employees of Crucell. The opinions in this manuscript are those of the authors and do not reflect the views of the U.S. Department of Defense. 
Despite the recent demonstration of partial HIV-1 vaccine efficacy in humans ${ }^{6}$, the immune responses required to protect against acquisition of infection have remained unclear.

Preclinical studies of HIV-1 vaccine candidates have begun to elucidate immunologic correlates of protection against neutralization-sensitive viruses ${ }^{1-3}$, but no study has to date reported vaccine protection against acquisition of heterologous, neutralization-resistant virus challenges ${ }^{1,7,8}$. Mucosal SIVmac251 infection of rhesus monkeys represents a stringent preclinical model of a highly pathogenic, neutralization-resistant virus swarm ${ }^{1,9,10}$, and repetitive mucosal challenges more closely mimic sexual HIV-1 transmission in humans than do single high-dose challenges ${ }^{10}$. We therefore performed two studies to evaluate the protective efficacy of optimized adenovirus/poxvirus and adenovirus/adenovirus vectorbased vaccines against repetitive, heterologous, intrarectal SIVmac251 challenges in rhesus monkeys.

In the first study, 40 Indian-origin rhesus monkeys (Macaca mulatta) that did not express the class I alleles Мати- $A * 01$, Мати- $B * 08$, and Мати- $B * 17$ associated with spontaneous virologic control ${ }^{11-13}$ were immunized by the intramuscular route with the following vaccine regimens expressing SIVsmE543 Gag-Pol and Env immunogens (N=8/group): (i) DNA prime, MVA boost; (ii) MVA prime, MVA boost; (iii) Ad26 prime, MVA boost; (iv) MVA prime, Ad26 boost; and (v) sham controls. Groups were balanced for susceptible and resistant TRIM5a alleles ${ }^{1,14}$. Monkeys were primed once at week 0 with $2 \times 10^{10}$ vp Ad26 vectors or $10^{8}$ pfu MVA vectors, or three times at weeks 0,4 , and 8 with $5 \mathrm{mg}$ DNA vaccines. Animals were then boosted once at week 24 with $2 \times 10^{10}$ vp Ad26 vectors or $10^{8}$ pfu MVA vectors.

The vaccine regimens elicited different profiles of cellular and humoral immune responses, as measured by IFN- $\gamma$ ELISPOT assays (Fig. 1a, Supplementary Fig. 1), multiparameter intracellular cytokine staining (ICS) assays ${ }^{8,15-17}$ (Fig. 1b, Supplementary Fig. 2), cellular immune breadth (Supplementary Fig. 3), SIVmac251 Env-specific binding antibody ELISAs (Fig. 1c), tier 1 neutralizing antibody (NAb) assays against tissue culture laboratory adapted (TCLA) tier 1 SIVsmE660 (CP3C-P-A8) and SIVmac251 (TCLA) pseudoviruses (Fig. 1d), and antibody-dependent cellular cytotoxicity (ADCC) and antibody-dependent cell-mediated virus inhibition (ADCVI) assays (Supplementary Fig. 4). Tier 2 NAb responses against neutralization-resistant SIVsmE660 (CR54-PK-2A5) and SIVmac251 (SIVmac251.30) pseudoviruses, however, were below the $50 \%$ neutralization cutoff for positivity, although positive trends were observed in all vaccinated groups (Supplementary Fig. 4).

To evaluate the protective efficacy of these vaccine regimens, all monkeys were challenged repetitively beginning at week 52 (six months following the boost immunization) with six intrarectal inoculations of the heterologous virus SIVmac251 utilizing a 1:1000 dilution (930 $\mathrm{TCID}_{50}$ ) of our challenge stock ${ }^{9}$. After the first challenge, $75 \%$ of sham control monkeys became infected, as compared with only $12-25 \%$ of the animals that received the heterologous vector regimens DNA/MVA, Ad26/MVA, and MVA/Ad26 (Fig. 1e). The percent uninfected animals declined proportionately with each challenge, and the majority of vaccinees and all controls were infected by the end of the challenge protocol. Monkeys that received the Ad26/MVA and MVA/Ad26 vaccines required 3 challenges to infect 50\% of animals in each group, whereas only 1 challenge was required to infect $50 \%$ of animals in 
the control group $(\mathrm{P}=0.004$ and $\mathrm{P}=0.006$, respectively, Wald tests, proportional hazard model). The heterologous vector regimens also exhibited decreased hazard ratios of 0.17 (CI $0.05-0.57$ ) to 0.20 (CI 0.06-0.63) as compared with the controls, corresponding to an 80$83 \%$ reduction in the per-exposure probability of infection (Fig. 1f; vaccine efficacy VE $=1$ - hazard ratio), utilizing the statistical approach of Self et al. ${ }^{4}$ and Gilbert et al. ${ }^{5}$. These data demonstrate vaccine protection against acquisition of infection following repetitive, heterologous, IR SIVmac251 challenges.

Control monkeys exhibited peak viral loads on day 14 following infection and then relatively stable mean setpoint viral loads of $5.85 \log$ SIV RNA copies/ml (Supplementary

Fig. 5). The Ad26/MVA and the MVA/Ad26 vaccines resulted, respectively, in at least 2.32 and $1.08 \mathrm{log}$ reductions of mean setpoint viral loads compared with sham controls for over 250 days ( $\mathrm{P}=0.0037$ for each vaccine versus sham, Wilcoxon rank-sum tests) (Fig. 1g, Supplementary Fig. 5). Moreover, half the animals in the Ad26/MVA group either demonstrated rapid and durable virologic control to undetectable levels (Fig. 1g; N=3) or remained uninfected (Fig. 1e; N=1). The Ad26/MVA and MVA/Ad26 vaccines also afforded a survival advantage as compared with the controls ( $\mathrm{P}=0.025$, log-rank test) (Supplementary Fig. 6).

We next evaluated the immunologic correlates of protection against acquisition of infection, defined as the number of challenges required to establish infection, and virologic control, defined as setpoint viral loads. Our pre-specified primary immunologic correlates analysis (Supplementary Table 1) demonstrated that protection against acquisition of infection was best correlated with Env binding ELISA antibody responses (Fig. 2a; P $<0.0001$, Spearman rank-correlation test) and tier $1 \mathrm{NAb}$ titers (Fig. $2 \mathrm{~b} ; \mathrm{P}=0.0034$ ) immediately prior to challenge. Protection against acquisition of infection also correlated with V2-specific antibodies that presumably represent a subset of total Env binding antibodies (Fig. 2e, f; $\mathrm{P}<0.0001$ ). Virologic control was correlated with Gag ELISPOT breadth (Fig. 2c; $\mathrm{P}=0.0002$ ) and magnitude (Fig. 2d; $\mathrm{P}=0.0058$ ) immediately prior to challenge, consistent with our previous observations ${ }^{18}$.

In our exploratory immunologic correlates analysis, we evaluated 35 humoral and cellular immune parameters at both peak and memory timepoints prior to challenge as possible immunologic correlates of acquisition and virologic control following challenge. No additional immune parameters were significantly correlated with protection against acquisition of infection in this analysis after multiple comparison adjustments (Supplementary Table 2). Gag-, Pol-, and Env-specific effector memory CD8+ T lymphocyte responses exhibited trends towards protection against acquisition but did not achieve statistical significance according to our pre-specified criteria. In contrast, multiple humoral and cellular immune responses were significantly correlated with virologic control (Supplementary Table 3), including Env ELISA, NAb, and ADCC responses as well as Gag ELISPOT magnitude and breadth, Pol ELISPOT magnitude, and Env CD4+ effector memory responses. These data suggest a model in which protection against acquisition of infection is correlated with vaccine-elicited Env-specific antibody responses, whereas virologic control may be correlated with both T lymphocyte and antibody responses. These distinct immunologic correlates likely reflect fundamentally different biologic requirements 
for blocking establishment of infection at the mucosal site of inoculation compared with controlling viral replication after infection has become disseminated ${ }^{19}$. However, the actual mechanisms of protection remain to be determined.

We next evaluated directly the hypothesis that Env was critical for blocking acquisition of infection in this system. In the second study, 40 rhesus monkeys that did not express the class I alleles Мати- $A * 01$, Мати- $B * 08$, and $М а т и-B * 17$ were immunized by the intramuscular route with $\mathrm{Ad} 35$ prime $^{20}, \mathrm{Ad} 26$ boost $^{21}$ vaccine regimens expressing (i) SIVsmE543 Gag-Pol (N=16); (ii) SIVsmE543 Gag-Pol and Env (N=16); and (iii) sham controls $(\mathrm{N}=8)$. Groups were balanced for susceptible and resistant TRIM5a alleles ${ }^{1,14}$. Monkeys were primed once at week 0 with $2 \times 10^{10} \mathrm{vp}$ Ad35 vectors and boosted once at week 24 with $2 \times 10^{10}$ vp Ad26 vectors. Cellular immune responses were assessed by IFN- $\gamma$ ELISPOT assays (Fig. 3a, Supplementary Fig. 7) and multiparameter ICS assays in both the periphery (Fig. 3b, Supplementary Fig. 8) and in colorectal mucosa (Supplementary Fig. 9). Env-specific humoral immune responses were assessed by ELISAs in both the periphery (Fig. 3c) and in colorectal mucosa (Supplementary Fig. 10), tier 1 NAb assays (Fig. 3d), and ADCC assays (Supplementary Fig. 11). Only marginal tier 2 NAb responses were observed (Supplementary Fig. 11).

We assessed protective efficacy of these vaccine regimens against repetitive, heterologous, intrarectal SIVmac251 challenges as described in the first study. After the first challenge, $50 \%$ of sham control monkeys became infected, as compared with only $12 \%$ of the animals that received the Gag-Pol-Env vaccine (Fig. 3e). The monkeys that received the Gag-PolEnv vaccine required 4 challenges to infect $50 \%$ of animals in each group, whereas only 1 challenge was required to infect $50 \%$ of animals in the control group (Fig. 3f; $\mathrm{P}=0.002$, Wald test, proportional hazard model). Moreover, the Gag-Pol-Env vaccine resulted in a decreased hazard ratio of 0.20 (CI $0.07-0.55$ ), corresponding to an $80 \%$ reduction in the perexposure probability of infection. In contrast, the Gag-Pol vaccine afforded only a marginal protective effect, demonstrating the critical role of Env in blocking acquisition of infection in this model. The Gag-Pol and Gag-Pol-Env vaccines resulted in, respectively, at least 1.59 $\log$ and 2.18 log reductions of setpoint viral loads as compared with controls (Fig. 3g, Supplementary Fig. 12; P=0.0006 and 0.0002, respectively, Wilcoxon rank-sum tests). Immunologic correlates of protection against acquisition of infection were consistent with the first study, and both peripheral (Fig. 4a-c) and rectal (Fig. 4d) Env-specific IgG correlated with reduced acquisition risk.

Taken together, these data demonstrate that optimized adenovirus/poxvirus and adenovirus/ adenovirus vector-based vaccines afforded significant protection against acquisition of infection following highly pathogenic, heterologous, neutralization-resistant SIVmac251 challenges in rhesus monkeys (Fig. 1e, 3e, Supplementary Fig. 13). Although several studies have previously shown partial protection against acquisition of neutralization-sensitive virus challenges ${ }^{1-3}$, no HIV-1 vaccine candidate has to date blocked acquisition of heterologous, difficult-to-neutralize virus challenges, including $\mathrm{Ad} 5^{7}$, DNA/Ad5 ${ }^{1}$, and $\mathrm{CMV}^{8}$ vaccines. In particular, a recent study demonstrated that a DNA/Ad5 vaccine afforded partial protection against acquisition of SIVsmE660, which is a neutralization-sensitive tier 1A virus in TZMbl neutralization assays, but the same vaccine afforded no efficacy against neutralization- 
resistant SIVmac251 ${ }^{1}$, highlighting important differences in the stringencies between these two SIV challenge models as well as potentially important phenotypic differences between Ad serotypes ${ }^{17}$. However, we note that the acquisition effect in the present study was relative rather than absolute and that the majority of vaccinees became infected by the end of the challenge protocol.

Our studies also demonstrate that inclusion of Env in the vaccine was required for the acquisition effect (Fig. 3e), despite an 18\% difference in the amino acid sequences between the vaccine strain and challenge virus. Moreover, our immunologic correlates analyses (Fig. 2, 4; Supplementary Tables 1-3) suggest that Env-specific antibodies may be critical for blocking acquisition of infection, whereas multiple cellular and humoral immune responses may correlate with virologic control, although the actual mechanisms of protection remain to be determined. In addition, the RV144 immunologic correlates analyses raised the hypothesis that vaccine-elicited V1/V2-specific antibodies may reduce HIV-1 acquisition risk in humans ${ }^{22}$. Our data (Fig. 2f, 4c) are consistent with this hypothesis, although it remains unclear whether $\mathrm{V} 2$-specific antibodies actually protect or simply represent a marker for other Env-specific antibodies or other protective factors.

Considerable efforts are currently underway to identify and to reverse engineer potent, broadly reactive monoclonal antibodies ${ }^{23,24}$. Although the induction of such NAb responses by a vaccine would be highly desirable, no Env immunogens have to date been developed that can elicit these responses. Our findings suggest that a substantial degree of protection can be achieved against stringent virus challenges even in the absence of high titers of tier 2 NAbs, perhaps reflecting the importance of antibody effector functions that may not be fully measured by traditional virus neutralization assays. Of note, the partial protection in the present study was observed with vectored Env and without a purified Env protein subunit boost. The degree to which an Env protein boost may further improve the protective efficacy afforded by these vaccine regimens remains to be determined.

In summary, our data demonstrate the first proof-of-concept that vaccination can protect against acquisition of stringent, heterologous, neutralization-resistant SIVmac251 challenges in rhesus monkeys. These findings, together with the observations of a critical requirement for Env and the distinct immunologic correlates of protection against acquisition of infection and virologic control, pave novel paths forward for HIV-1 vaccine development.

\section{Methods Summary}

For each study, 40 Indian-origin rhesus monkeys (Macaca mulatta) were vaccinated with DNA, MVA ${ }^{25}, \mathrm{Ad}_{26}{ }^{21}$, or Ad35 ${ }^{20}$ expressing SIVsmE543 Gag-Pol and/or Env antigens or received a sham vaccine. Cellular immune responses were measured by IFN- $\gamma$ ELISPOT $^{18}$, multiparameter intracellular cytokine staining (ICS) ${ }^{8,15-17}$, and epitope mapping ${ }^{26}$ assays. Humoral immune responses were measured by Env ELISA ${ }^{27}$, TZM-bl pseudovirus neutralizing antibody $(\mathrm{NAb})^{28}$, antibody-dependent cellular cytotoxicity (ADCC $)^{29}$, and antibody-dependent cell-mediated virus inhibition (ADCVI) ${ }^{30}$ assays. Six months following the boost immunization, all monkeys received six challenges by the intrarectal route with the fully heterologous, neutralization-resistant virus SIVmac251 utilizing a 1:1000 dilution (930 
$\mathrm{TCID}_{50}$ ) of the challenge stock ${ }^{9}$. Protective efficacy was determined by resistance to acquisition of infection, defined as the number of challenges required to establish infection, and virologic control, defined as setpoint viral loads. Immunologic correlates of protection against acquisition of infection and virologic control were evaluated by pre-specified primary and exploratory analyses.

\section{Methods}

\section{Animals, immunizations, and challenges}

80 Indian-origin, outbred, young adult, male and female, specific pathogen-free (SPF) rhesus monkeys (Macaca mulatta) that did not express the class I alleles Mamu- $A * 01$, Мати- $B * 08$, and $М а т и-B * 17$ associated with spontaneous virologic control ${ }^{11-13}$ were housed at New England Primate Research Center (NEPRC), Southborough, MA. 40 animals were utilized for each study. Groups were balanced for susceptible and resistant TRIM5a alleles ${ }^{1,14}$. Immunizations were performed by the intramuscular route in the quadriceps muscles with $2 \times 10^{10}$ vp Ad35 vectors ${ }^{20}, 2 \times 10^{10}$ vp Ad26 vectors ${ }^{21}, 10^{8}$ pfu MVA vectors $^{25}$, or $5 \mathrm{mg}$ DNA vaccines expressing SIVsmE543 Gag-Pol and/or Env gp140. Monkeys were primed at week 0 and boosted at week 24, except DNA vaccine priming that was performed at weeks 0,4 , and 8 . To evaluate for protective efficacy and immunologic correlates, all monkeys were challenged repetitively beginning at week 52 with six intrarectal inoculations of the heterologous virus SIVmac251 utilizing a 1:1000 dilution (930 $\mathrm{TCID}_{50}$ ) of our challenge stock ${ }^{9}$. Monkeys were bled weekly for viral loads (Siemans Diagnostics), and the date of infection was defined as the last challenge timepoint prior to the first positive SIV RNA level. Animals were followed to determine setpoint viral loads. All animal studies were approved by the Harvard Medical School Institutional Animal Care and Use Committee (IACUC).

\section{Cellular immune assays}

SIV-specific cellular immune responses were assessed by IFN- $\gamma$ ELISPOT assays ${ }^{18}$ and multiparameter intracellular cytokine staining (ICS) assays ${ }^{8,15-17}$ essentially as described. ELISPOT assays utilized pools of SIVsmE543 and SIVmac239 Gag, Pol, and Env peptides. Analyses of cellular immune breadth utilized subpools of 10 peptides covering each antigen. Peptides were 15 amino acids in length and overlapped by 11 amino acids. 9-color ICS assays utilized mAbs (Becton-Dickinson) against CD3 (SP34; Alexa700), CD4 (L200; AmCyan), CD8 (SK1; allophycocyanin-cyanine7 [APC-Cy7]), CD28 (L293; peridinin chlorophyll-Acyanine5.5 [PerCP-Cy5.5]), CD95 (DX2; phycoerythrin [PE]), CD69 (TP1.55.3; phycoerythrin-Texas Red [energy coupled dye; ECD]; Beckman Coulter), IFN- $\gamma$ (B27; phycoerythrin-cyanine7 [PE-Cy7]), IL-2 (MQ1-17H12; allophycocyanin [APC]) and TNF-a (Mab11; fluorescein isothiocya [FITC]). IFN- $\gamma$ backgrounds were consistently $<0.01 \%$ in PBMC and $<0.05 \%$ in colorectal biopsy specimens.

\section{Humoral immune assays}

SIV-specific humoral immune responses were assessed by SIVmac251 Env ELISAs ${ }^{27}$, TZM-bl luciferase-based virus neutralization assays ${ }^{28}$ against tier 1 SIVsmE660 (CP3C-PA8) and SIVmac251 (TCLA) pseudoviruses, TZM-bl virus neutralization assays against tier 
2 SIVsmE660 (CR54-PK-2A5) and SIVmac251 (SIVmac251.30) pseudoviruses, antibodydependent cellular cytotoxicity (ADCC) assays ${ }^{29}$, and antibody-dependent cell-mediated virus inhibition (ADCVI) assays ${ }^{30}$. V2 binding assays were performed by surface plasmon resonance with a Biacore 2000 or T200 using a 1:50 serum dilution and a cyclic SIVsmE543 $\mathrm{V} 2$ peptide containing an $\mathrm{N}$-terminal biotin tag (CIKNNSCAGLEQEPMIGCKFNMTGLKRDKKIEYNETWYSRDLICEQPANGSESKCY ) and immobilized on streptavidin-coated CM5 chips. Mucosal antibodies were assessed using rectal secretions collected with Weck-Cel sponges. Approximately $100 \mathrm{ul}$ rectal secretions were eluted and diluted 6-fold, and total IgG and IgA as well as SIV Env-specific IgG and IgA (Immune Technology Corporation) were measured by ELISA using a biotinconjugated anti-monkey IgG and IgA (Alpha Diagnostics) secondary antibody. Mucosal titers were defined as endpoint ELISA titers multiplied by the dilution of the eluted secretions. Samples exhibited comparable levels of total IgG.

\section{Statistical analyses and immunologic correlates}

Protection against acquisition of infection was analyzed using Wald tests with a proportional hazard model and the exact conditional likelihood method for breaking ties. A discrete time model provided similar estimates. The number of challenges required for $50 \%$ infection of each group, hazard ratios with $95 \%$ confidence intervals (CI), per-exposure vaccine efficacy (VE), and per-exposure risks of infection were quantitated. VE was defined as the reduction in the per-exposure probability of infection as previously described ${ }^{4,5}$. Analyses of virologic and immunologic data were performed by Wilcoxon rank-sum tests and analysis of survival by log-rank tests. For these tests, $\mathrm{P}<0.05$ was considered significant, and two-tailed tests were performed. Immunologic correlates were evaluated by a focused primary analysis and a detailed exploratory analysis using Spearman rank-correlation tests. In the primary analysis, $\mathrm{P}<0.01$ was considered significant, whereas in the exploratory analysis, $\mathrm{P}<0.0014$ was considered significant to adjust for multiple comparisons.

\section{Supplementary Material}

Refer to Web version on PubMed Central for supplementary material.

\section{Acknowledgements}

We thank M. Pensiero, A. Fauci, E. Borducchi, S. Clark, R. Hamel, S. King, P. Kozlowski, A. La Porte, G. Landucci, A. Oza, J. Perry, L. Peter, A. Riggs, G. Shaw, N. Simmons, K. Smith, K. Stanley, F. Stephens, Y.-H. Sun, G. Weverling, and E. Zablowsky for generous advice, assistance, and reagents. We thank L. Picker, G. Silvestri, and B. Walker for critically reviewing this manuscript. The SIVmac239 peptide pools were obtained from the NIH AIDS Research and Reference Reagent Program. We acknowledge support from the U.S. Military Research and Material Command and the U.S. Military HIV Research Program (W81XWH-07-2-0067); the Ragon Institute of MGH, MIT, and Harvard; the National Institutes of Health (AI066924, AI078526, AI084794, AI095985, AI060354, AI002642, RR000168); and the Bill and Melinda Gates Foundation.

\section{References}

1. Letvin NL, et al. Immune and Genetic Correlates of Vaccine Protection Against Mucosal Infection by SIV in Monkeys. Sci Transl Med. 2011; 3:81ra36. 
2. Lai L, et al. Prevention of infection by a granulocyte-macrophage colony-stimulating factor coexpressing DNA/modified vaccinia Ankara simian immunodeficiency virus vaccine. J Infect Dis. 2011; 204:164-173. [PubMed: 21628671]

3. Barnett SW, et al. Antibody-mediated protection against mucosal simian-human immunodeficiency virus challenge of macaques immunized with alphavirus replicon particles and boosted with trimeric envelope glycoprotein in MF59 adjuvant. J Virol. 2010; 84:5975-5985. [PubMed: 20392857]

4. Hudgens MG, et al. Power to detect the effects of HIV vaccination in repeated low-dose challenge experiments. J Infect Dis. 2009; 200:609-613. [PubMed: 19591571]

5. Hudgens MG, Gilbert PB. Assessing vaccine effects in repeated low-dose challenge experiments. Biometrics. 2009; 65:1223-1232. [PubMed: 19397589]

6. Rerks-Ngarm S, et al. Vaccination with ALVAC and AIDSVAX to prevent HIV-1 infection in Thailand. N Engl J Med. 2009; 361:2209-2220. [PubMed: 19843557]

7. Wilson NA, et al. Vaccine-induced cellular immune responses reduce plasma viral concentrations after repeated low-dose challenge with pathogenic simian immunodeficiency virus SIVmac239. J Virol. 2006; 80:5875-5885. [PubMed: 16731926]

8. Hansen SG, et al. Profound early control of highly pathogenic SIV by an effector memory T-cell vaccine. Nature. 2011; 473:523-527. [PubMed: 21562493]

9. Liu J, et al. Low-dose mucosal simian immunodeficiency virus infection restricts early replication kinetics and transmitted virus variants in rhesus monkeys. J Virol. 2010; 84:10406-10412. [PubMed: 20686016]

10. Keele BF, et al. Low-dose rectal inoculation of rhesus macaques by SIVsmE660 or SIVmac251 recapitulates human mucosal infection by HIV-1. J Exp Med. 2009; 206:1117-1134. [PubMed: 19414559]

11. Yant LJ, et al. The high-frequency major histocompatibility complex class I allele Mamu-B*17 is associated with control of simian immunodeficiency virus SIVmac239 replication. J Virol. 2006; 80:5074-5077. [PubMed: 16641299]

12. Mothe BR, et al. Expression of the major histocompatibility complex class I molecule Mamu-A*01 is associated with control of simian immunodeficiency virus SIVmac239 replication. J Virol. 2003; 77:2736-2740. [PubMed: 12552014]

13. Loffredo JT, et al. Mamu-B*08-positive macaques control simian immunodeficiency virus replication. J Virol. 2007; 81:8827-8832. [PubMed: 17537848]

14. Lim SY, et al. TRIM5alpha Modulates Immunodeficiency Virus Control in Rhesus Monkeys. PLoS Pathog. 2010; 6:e1000738. [PubMed: 20107597]

15. Okoye A, et al. Progressive CD4+ central memory T cell decline results in CD4+ effector memory insufficiency and overt disease in chronic SIV infection. The Journal of experimental medicine. 2007; 204:2171-2185. [PubMed: 17724130]

16. Pitcher CJ, et al. Development and homeostasis of T cell memory in rhesus macaque. J Immunol. 2002; 168:29-43. [PubMed: 11751943]

17. Liu J, et al. Magnitude and phenotype of cellular immune responses elicited by recombinant adenovirus vectors and heterologous prime-boost regimens in rhesus monkeys. J Virol. 2008; 82:4844-4852. [PubMed: 18337575]

18. Liu J, et al. Immune control of an SIV challenge by a T-cell-based vaccine in rhesus monkeys. Nature. 2009; 457:87-91. [PubMed: 18997770]

19. Haase AT. Targeting early infection to prevent HIV-1 mucosal transmission. Nature. 2010; 464:217-223. [PubMed: 20220840]

20. Vogels R, et al. Replication-deficient human adenovirus type 35 vectors for gene transfer and vaccination: efficient human cell infection and bypass of preexisting adenovirus immunity. $\mathrm{J}$ Virol. 2003; 77:8263-8271. [PubMed: 12857895]

21. Abbink P, et al. Comparative seroprevalence and immunogenicity of six rare serotype recombinant adenovirus vaccine vectors from subgroups B and D. J Virol. 2007; 81:4654-4663. [PubMed: 17329340]

22. Haynes, B. AIDS Vaccine 2011. Bangkok, Thailand: 2011. Case control study of the RV144 trial for immune correlates: the analysis and way forward. 
23. Walker LM, et al. Broad and potent neutralizing antibodies from an African donor reveal a new HIV-1 vaccine target. Science. 2009; 326:285-289. [PubMed: 19729618]

24. Wu X, et al. Rational design of envelope identifies broadly neutralizing human monoclonal antibodies to HIV-1. Science. 2010; 329:856-861. [PubMed: 20616233]

25. Ourmanov I, et al. Comparative efficacy of recombinant modified vaccinia virus Ankara expressing simian immunodeficiency virus (SIV) Gag-Pol and/or Env in macaques challenged with pathogenic SIV. J Virol. 2000; 74:2740-2751. [PubMed: 10684290]

26. Barouch DH, et al. Mosaic HIV-1 vaccines expand the breadth and depth of cellular immune responses in rhesus monkeys. Nat Med. 2010; 16:319-323. [PubMed: 20173752]

27. Nkolola JP, et al. Breadth of neutralizing antibodies elicited by stable, homogeneous clade A and clade C HIV-1 gp140 envelope trimers in guinea pigs. J Virol. 2010; 84:3270-3279. [PubMed: 20053749]

28. Montefiori, D. Evaluating neutralizing antibodies against HIV, SIV and SHIV in luciferase reporter gene assays. Current Protocols in Immunology. John Wiley \& Sons; 2004.

29. Ferrari G, et al. A HIV-1 gp120 Envelope Human Monoclonal Antibody that Recognizes a C1 Conformational Epitope Mediates Potent ADCC Activity and Defines a Common ADCC Epitope in Human HIV-1 Serum. J Virol. 2011

30. Forthal DN, Landucci G, Daar ES. Antibody from patients with acute human immunodeficiency virus (HIV) infection inhibits primary strains of HIV type 1 in the presence of natural-killer effector cells. Journal of virology. 2001; 75:6953-6961. [PubMed: 11435575] 

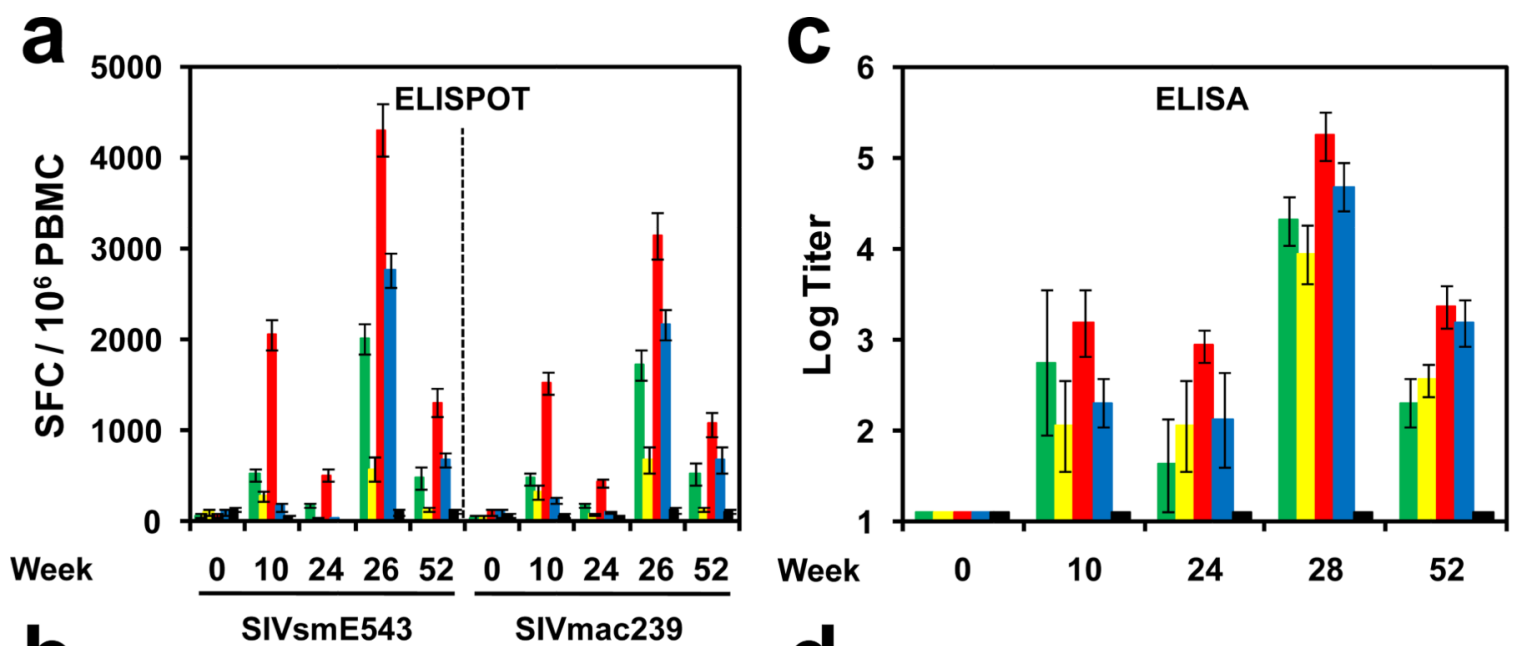

b

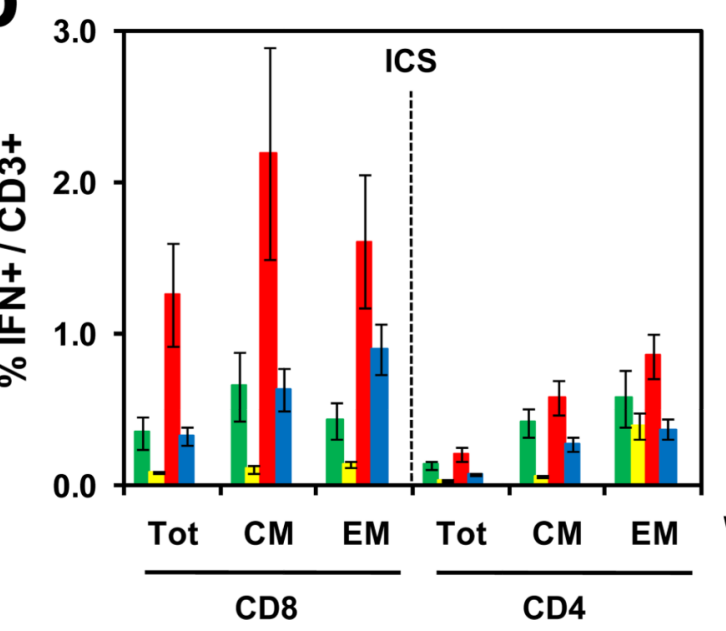

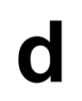

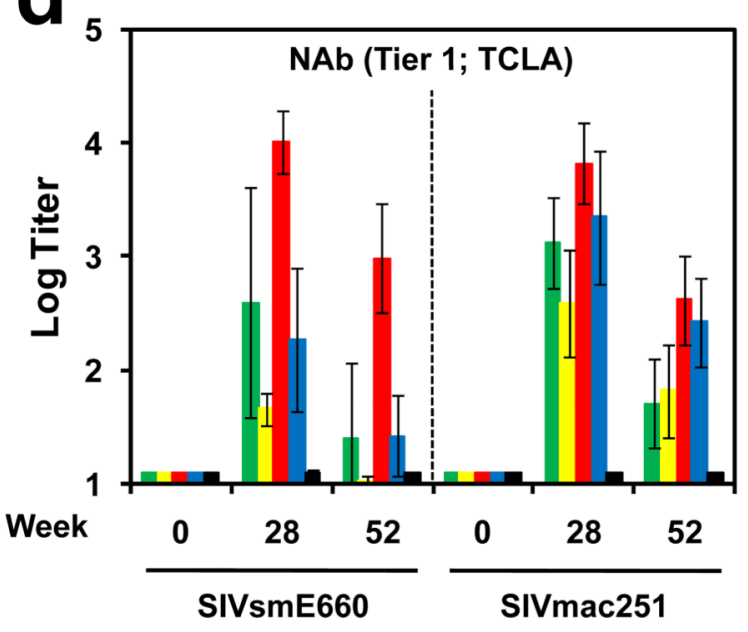

- DNA/MVA MVA/MVA - Ad26/MVA MVA/Ad26

- Sham 


\begin{tabular}{|l|l|l|l|l|l|l|}
\hline & \\
\hline
\end{tabular}

Figure 1. Immunogenicity and protective efficacy of the adenovirus/poxvirus vaccines a, Cellular immune responses to SIVsmE543 and SIVmac239 Gag, Pol, and Env as determined by IFN- $\gamma$ ELISPOT assays at weeks 0, 10, 24, 26, and 52. b, CD8+ and CD4+ total, central/transitional memory (CM; CD28+CD95+), and effector memory (EM; CD28CD95+) responses to Gag, Pol, and Env as determined by multiparameter IFN- $\gamma$ ICS assays at week 26. c, SIVmac251 Env ELISAs at weeks 0, 10, 24, 28, and 52. d, SIVsmE660 and SIVmac251 tier 1 pseudovirus NAb assays at weeks 0, 28, and 52. Error bars reflect s.e.m. e, Number of challenges required for acquisition of infection in each vaccine group. $\mathbf{f}$, Statistical analyses include the number of challenges required for 50\% infection, hazard ratios with $95 \%$ confidence intervals (CI), per-exposure vaccine efficacy (VE), and perexposure risks of infection in each group. P-values reflect Wald tests using a proportional hazard model. g, Log SIV RNA copies/ml are depicted for each vaccine group at viral setpoint (day 84 ). $* * \mathrm{P}=0.0037$, Wilcoxon rank-sum tests. The horizontal lines reflect mean setpoint log viral loads. 

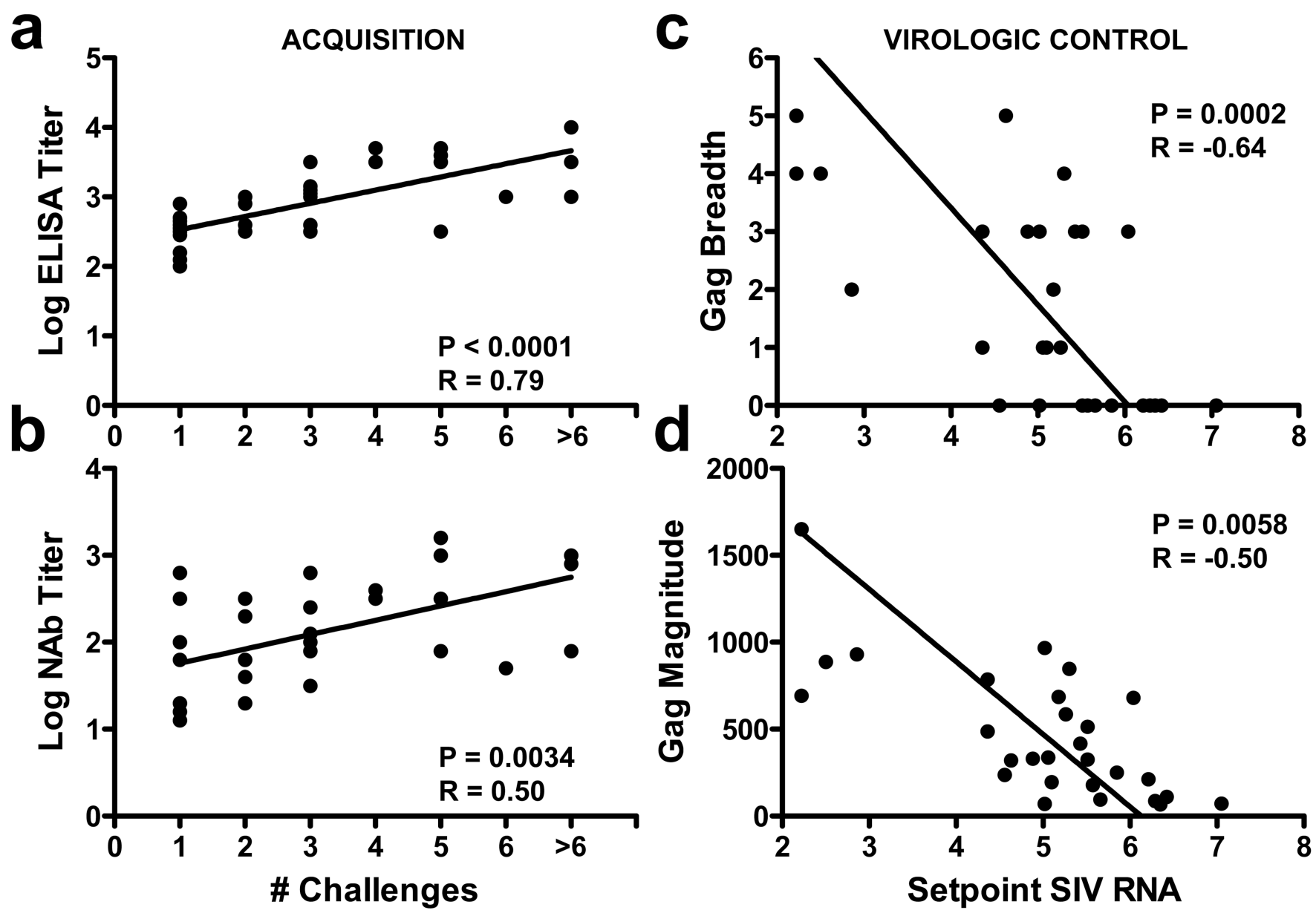

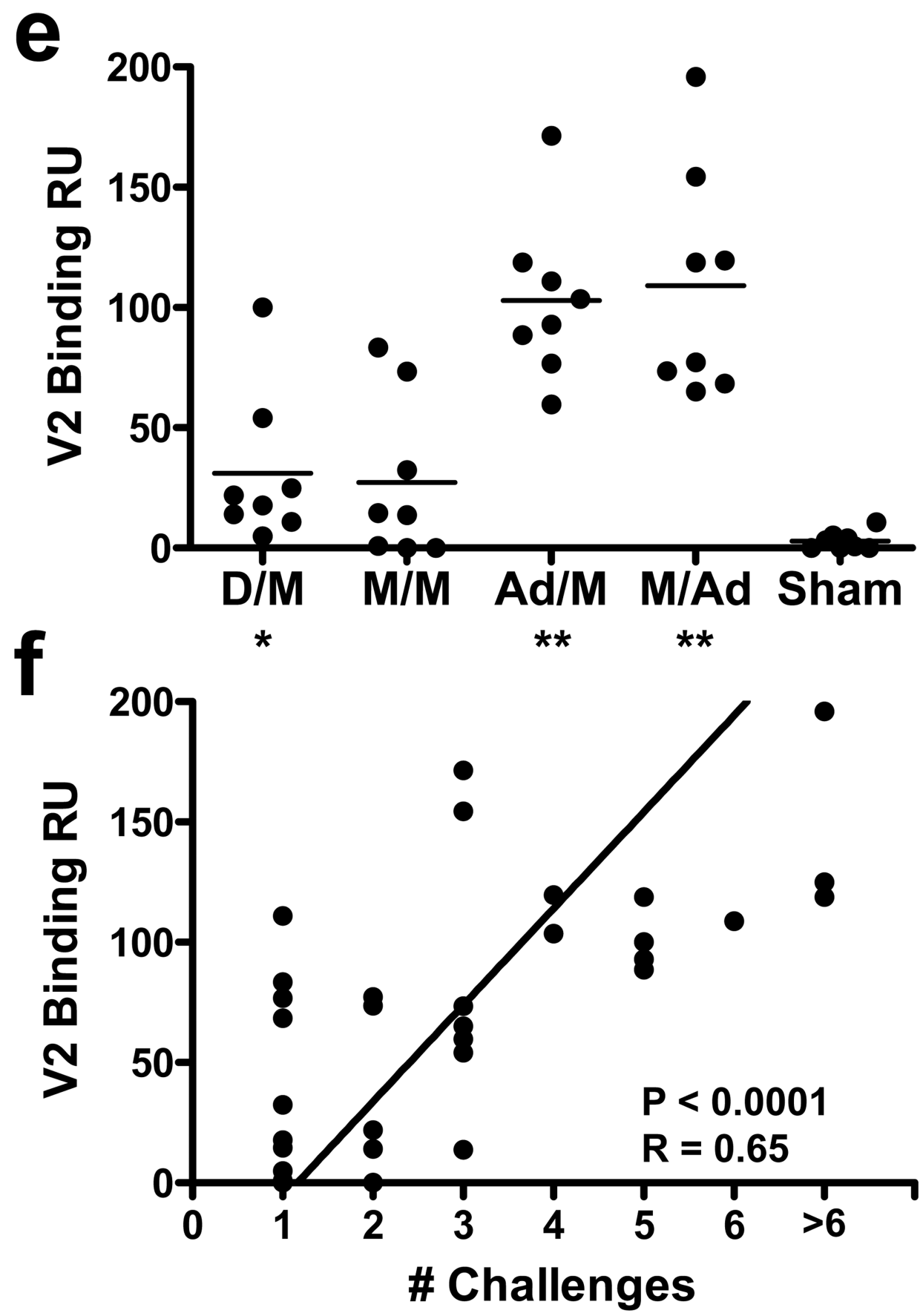

Figure 2. Correlates of protection against acquisition of infection and virologic control with the adenovirus/poxvirus vaccines

Correlation of (a) $\log$ ELISA titers immediately prior to challenge and (b) log tier $1 \mathrm{NAb}$ titers immediately prior to challenge with the number of challenges required to establish infection. Correlation of (c) Gag ELISPOT breadth prior to challenge and (d) Gag ELISPOT magnitude prior to challenge with setpoint viral loads following challenge. Correlates analyses included the 32 vaccinated monkeys (a, b) or the 29 vaccinated animals that became infected $(\mathbf{c}, \mathbf{d})$ and did not include the sham controls. P-values reflect Spearman 
rank-correlation tests. e, V2-specific binding antibodies assessed by surface plasmon resonance response units $(\mathrm{RU})$ for each vaccine group at week $30 . * \mathrm{P}=0.002$, ** $\mathrm{P}=0.0007$, Wilcoxon rank-sum tests. The horizontal lines reflect mean responses. f, Correlation of V2specific antibody responses with the number of challenges required to establish infection. 


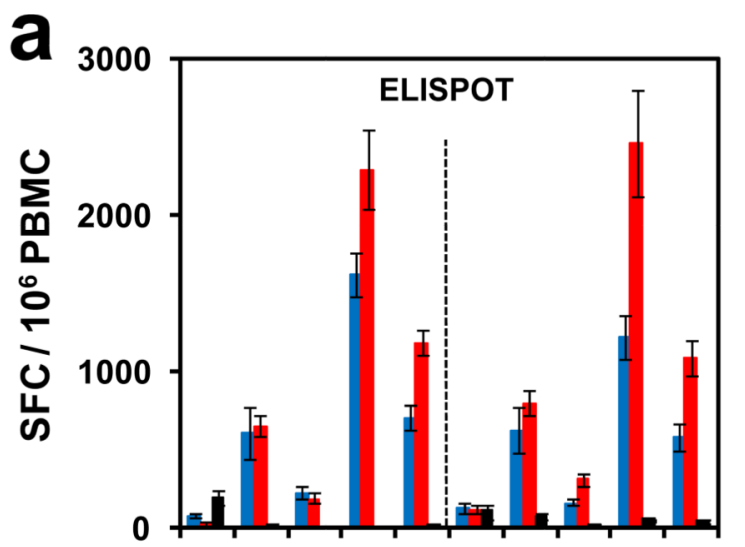

C.

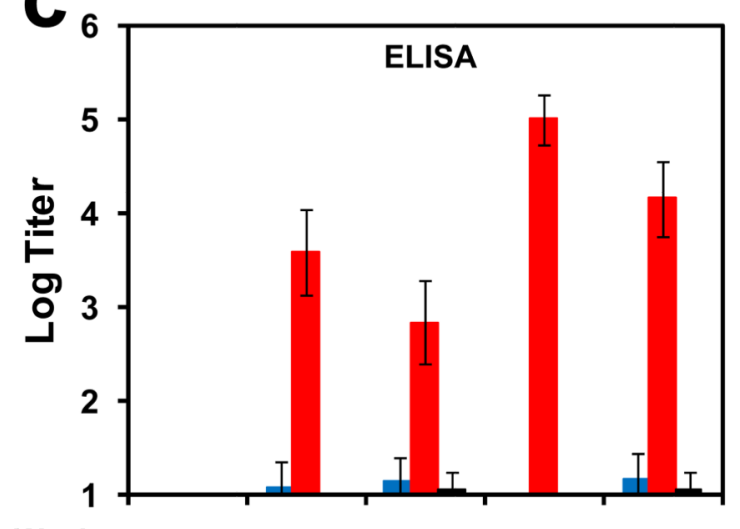

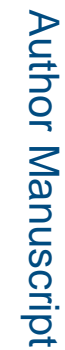
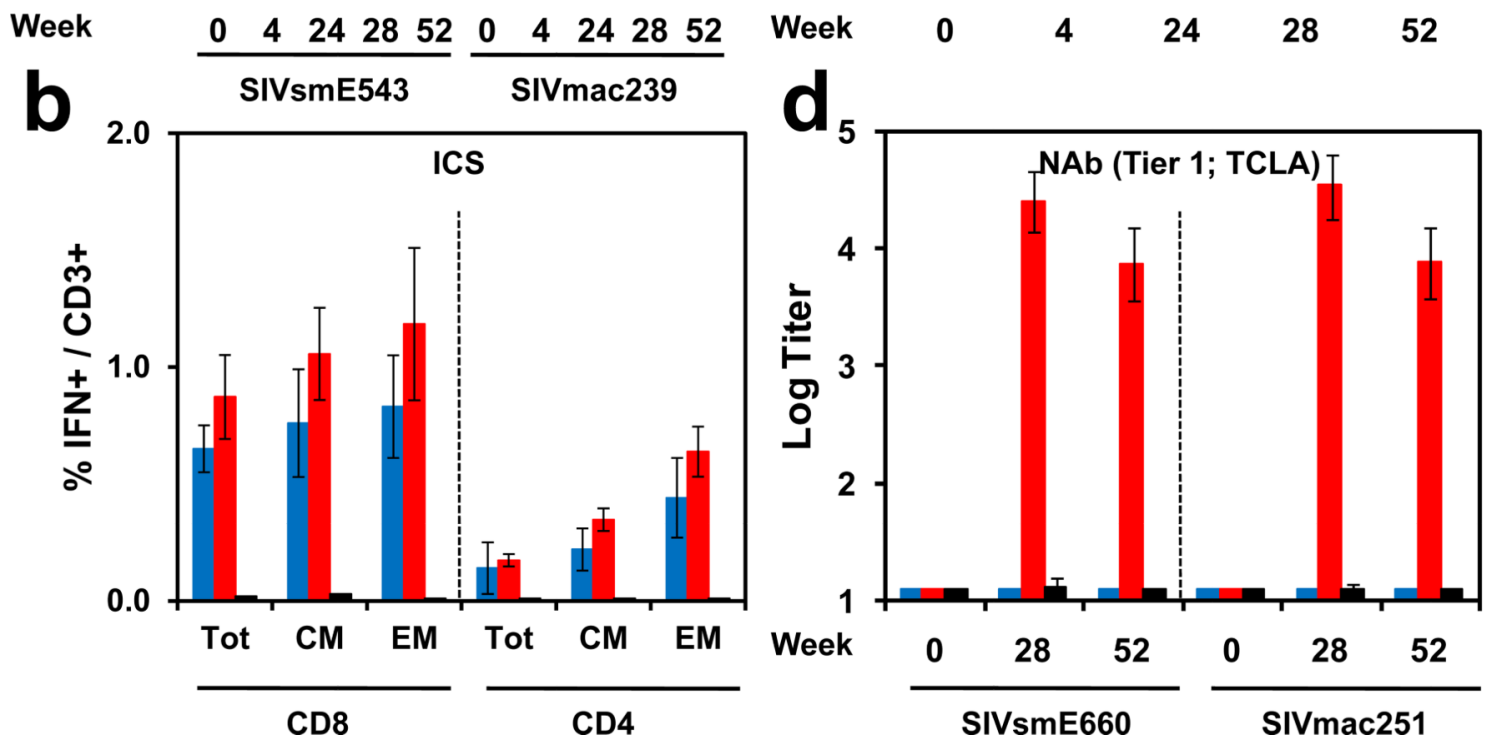

- Gag-Pol
- Gag-Pol-Env

- Sham

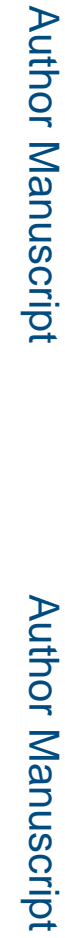



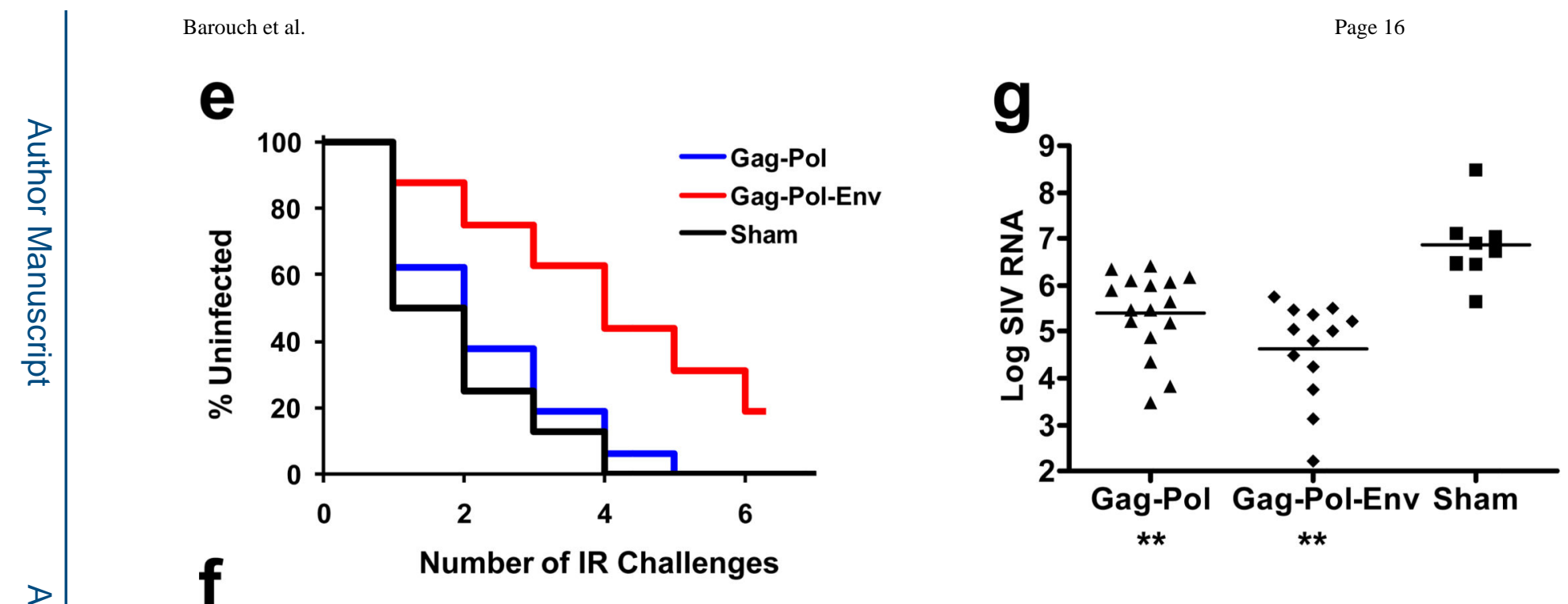

\begin{tabular}{|l|l|l|l|l|l|}
\hline & $\begin{array}{l}\text { \# Challenges for } \\
50 \% \text { Infection }\end{array}$ & $\begin{array}{l}\text { P-Value } \\
\text { vs Sham* }\end{array}$ & $\begin{array}{l}\text { Hazard Ratio } \\
(95 \% \mathrm{Cl})\end{array}$ & $\begin{array}{l}\text { Per-Exposure } \\
\text { Vaccine Efficacy }\end{array}$ & $\begin{array}{l}\text { Per-Exposure } \\
\text { Risk of Infection }\end{array}$ \\
\hline Gag-Pol & 2 & 0.46 & $0.71(0.29-1.74)$ & $29 \%$ & 0.44 \\
\hline Gag-Pol-Env & 4 & 0.002 & $0.20(0.07-0.55)$ & $80 \%$ & 0.20 \\
\hline Sham & 1 & N/A & 1.0 & N/A & 0.53 \\
\hline & * Wald test, proportional hazard model & \\
\end{tabular}

Figure 3. Immunogenicity and protective efficacy of the adenovirus/adenovirus vaccines a, Cellular immune responses to SIVsmE543 and SIVmac239 Gag, Pol, and Env as determined by IFN- $\gamma$ ELISPOT assays at weeks 0, 4, 24, 28, and 52. b, CD8+ and CD4+ total, central/transitional memory (CM; CD28+CD95+), and effector memory (EM; CD28CD95+) responses to Gag, Pol, and Env as determined by multiparameter IFN- $\gamma$ ICS assays at week 28. c, SIVmac251 Env ELISAs at weeks 0, 4, 24, 28, and 52. d, SIVsmE660 and SIVmac251 tier 1 pseudovirus NAb assays at weeks 0, 28, and 52. Error bars reflect s.e.m. e, Number of challenges required for acquisition of infection in each vaccine group. $\mathbf{f}$, Statistical analyses include the number of challenges required for $50 \%$ infection, hazard ratios with $95 \%$ confidence intervals (CI), per-exposure vaccine efficacy (VE), and perexposure risks of infection in each group. P-values reflect Wald tests using a proportional hazard model. g, Log SIV RNA copies/ml are depicted for each vaccine group at viral setpoint (day 84). ** $\mathrm{P}<0.001$, Wilcoxon rank-sum tests. The horizontal lines reflect mean setpoint log viral loads. 

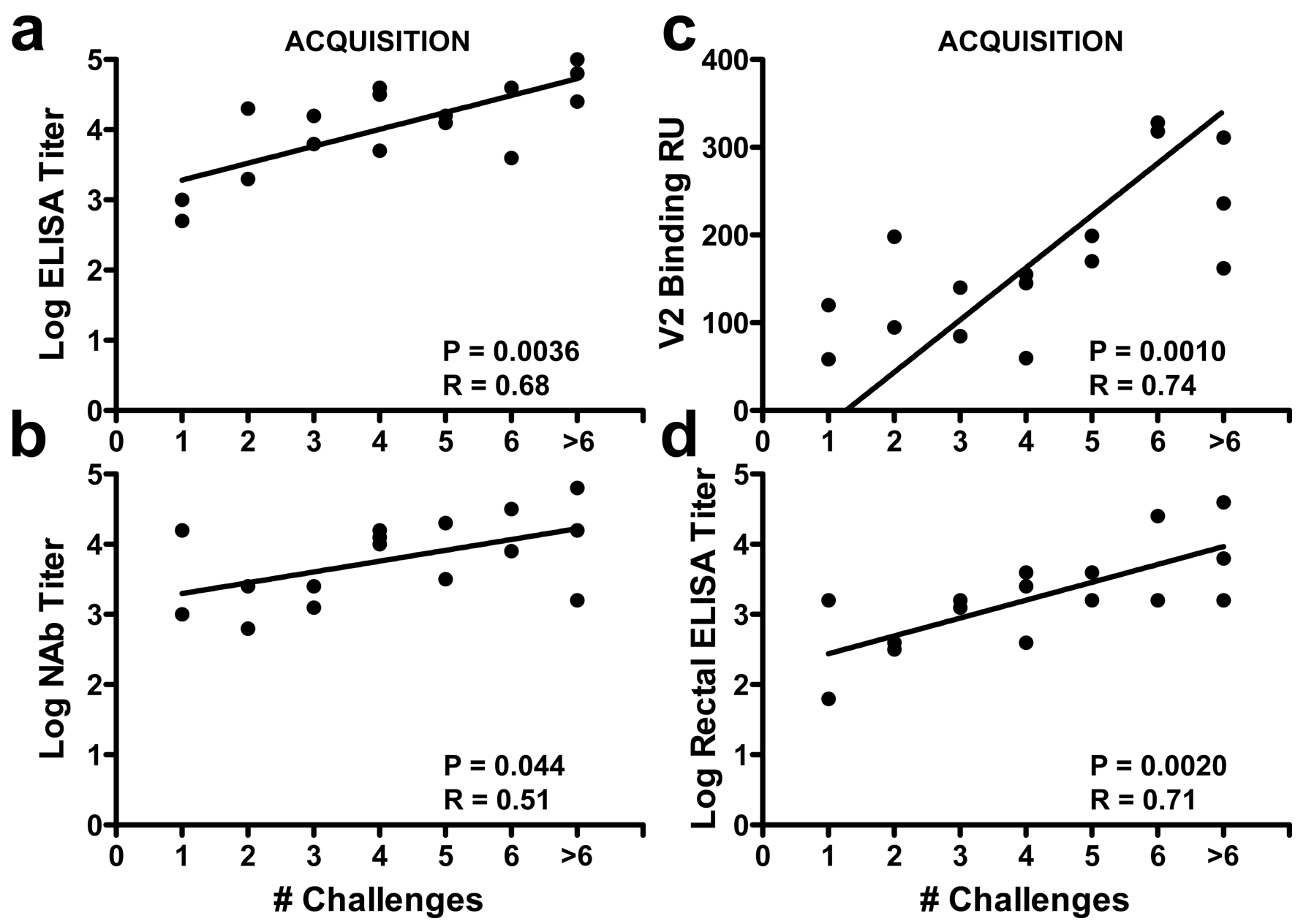

Figure 4. Correlates of protection against acquisition of infection with the adenovirus/adenovirus vaccines

Correlation of (a) log ELISA titers immediately prior to challenge, (b) log tier 1 NAb titers immediately prior to challenge, (c) V2-specific antibody responses, and (d) rectal IgG antibody responses with the number of challenges required to establish infection. Correlates analyses included the 16 Gag-Pol-Env vaccinated monkeys and did not include the Gag-Pol vaccinated monkeys or the sham controls. P-values reflect Spearman rank-correlation tests. 